\title{
Trampolines in New Zealand: a decade of injuries
}

\author{
David J. Chalmers PhD*, Patria A. Hume MSc Hons ${ }^{\dagger}$ and Barry D. Wilson PhD ${ }^{\dagger}$ \\ *Injury Prevention Research Unit and ${ }^{\dagger}$ School of Physical Education, University of Otago, Dunedin, New \\ Zealand
}

\begin{abstract}
Despite international concern about the safety of trampolines, they have become increasingly popular in New Zealand. While internationally attention has centred on a relatively few cases of catastrophic cervical spine injury, little research effort has been directed at placing these incidents in a wider context. To redress this, a descriptive epidemiological study of trampoline-related injury in New Zealand was undertaken. National hospitalization and mortality data for a 10-year period revealed 2098 hospitalizations and two deaths. The incidence rate for hospitalizations increased from 3.1 per 100000 population per year in 1979 to 9.3 in 1988. Of the hospitalized victims, $71 \%$ were injured on home trampolines and $80 \%$ fell from the trampoline to the surrounding surface. Fractures were the commonest type of injury (68\%), and the body site most frequently involved was the upper limb (53\%). There was no evidence of a high incidence of severe head and neck injuries. It was concluded that, although existing trampoline standards addressed many of the issues raised by this reasearch, measures to reduce the impact of falls from trampolines to the ground and to prohibit the provision of trampolines as 'play equipment' are required.
\end{abstract}

Keywords: Trampoline, recreation, epidemiology, prevention, standard

The trampoline has been the subject of controversy almost ever since it was patented in 1936 by George Nissen. Described as a 'rebound device activated by vertical jumping ${ }^{\prime 1}$, the trampoline was used in the training of fighter pilots during the Second World War, after which it became increasingly popular in Europe and North America as an item of gymnastic equipment. Its popularity declined, however, in the wake of widespread publicity given to reports of severe head and neck injuries being sustained by users of the equipment, predominantly when landing in the centre of the trampoline bed ${ }^{2-4}$. While many writers have called for the banning or restricted use of the trampoline $e^{2-7}$, others have provided a spirited defence of the equipment ${ }^{8-10}$, and in some instances opinion appears to have softened over time ${ }^{11}$.

Efforts to reduce the risk of injury to trampolinists have included the publication of a safety standard, ASTM F $381^{1}$, the development of guidelines for the safe use of the trampoline ${ }^{12-14}$, the development of

Address for correspondence: Dr David J Chalmers, Injury Prevention Research Unit, Department of Preventive and Social Medicine, Medical School, University of Otago, PO Box 913, Dunedin, New Zealand

(C) 1994 Butterworth-Heinemann Ltd 0306-3674/94/040234-05 instructional methods and programmes ${ }^{15,16}$, and the introduction of accreditation programmes for instructors ${ }^{17-19}$.

While considerable attention has been focused on a relatively small number of cases of catastrophic injury to the cervical spine, only two studies reported in refereed journals have adopted a broader epidemiological perspective in examining the problem of trampoline-related injury ${ }^{2,7}$. These studies have shown that home trampolines are commonly involved; that while falls onto the bed of the trampoline are the most common cause of injury, falls from the trampoline occur comparatively frequently; and that injuries to the extremities are more common than those to the spine. Although the cases in these studies were identified from emergency department records, suggesting lower levels of injury severity than for the cases referred to above, both included more serious injuries requiring hospital admission.

In New Zealand trampolines are used competitively by relatively few people but are popular for recreation, particularly among children and adolescents. It has been estimated that as many as 40000 trampolines are in regular use in this country of only 3.5 million people ${ }^{20}$. Concern at the risk of injury associated with trampoline use was first raised in New Zealand by Benis ${ }^{21}$, and more recently Chalmers and Langley 22 have reported that approximately $20 \%$ of injuries related to playground equipment and resulting in hospitalization can be attributed to trampolines. In response to calls from these authors for the introduction of safety standards for the design, construction and use of trampolines, the Standards Association of New Zealand has recently modified the American Society for Testing and Materials trampoline standard ASTM F381-84 ${ }^{1}$, which has been designated NZS 5855:199323.

The present study was undertaken because of concern that while much had been written about the dangers of trampolines, little sound epidemiological research has been reported on which to base recommendations regarding this equipment. The present paper is one of three arising from this study. An analysis of emergency department data is reported elsewhere ${ }^{24}$, as is a review of the debate over this equipment ${ }^{20}$.

\section{Methods}

National hospital discharge and mortality databases were searched for cases associated with trampolines. The source of discharge data was the Ministry of 
Health's New Zealand Health Information Service (NZHIS) public hospital morbidity data files for the period 1979-1988. The NZHIS records data on all discharges from public hospitals (for convenience, the term 'hospitalization' will be used to refer to 'hospital discharges'). Public hospitals treat the great majority of acute injury cases. Injuries are coded according to the World Health Organization's International Classification of Diseases (ICD) ${ }^{25}$ Injury and Poisoning ( $\mathrm{N}$ codes). Those cases with a principal diagnosis in the range N800-999 were included in the present study. Readmissions were excluded.

The circumstances of injury are coded on the morbidity file according to the ICD ${ }^{25}$ Supplementary Classification of External Causes of Injury and Poisoning (E codes). In addition, the morbidity file contains free-text descriptions of the circumstances of injury ( 30 characters) and the place of occurrence (12 characters). The search for cases of trampolinerelated injury began with the selection of all cases on the morbidity file that had a relevant $\mathrm{E}$ code, being $880-888,916-918$, 920, 927, 928, or 986-988. The free-text descriptions of the circumstances of injury for these cases were then searched electronically for mention of the words 'tramp', 'rebound' and 'jogger', and those cases clearly identified as involving a trampoline were retained.

Coding frames developed for the project were used to classify further the type and body site of the injuries, and the circumstances and place of occurrence of the incidents. Since no specific measure of injury severity is recorded by the NZHIS, the cases identified from the 1988 morbidity file were assigned Abbreviated Injury Scale (AIS) ${ }^{26}$ severity scores, using the ICDMAP program developed by McKenzie et al. ${ }^{27}$. This program translates ICD N codes into AIS severity scores, which range from 1 (minor) to 6 (virtually unsurvivable). 1988 was the first year in which data were in a form that was suitable for translation.

The source of mortality data was the NZHIS mortality files for the period 1978-1987. As for the hospitalization files, the circumstances of injury are coded according to the $I C D^{25} \mathrm{E}$ codes and there are free-text descriptions of the nature and circumstances (95 characters). The search for cases followed the procedure described above. Since the nature of injury resulting in death is not coded in the NZHIS mortality files, this was obtained from the free-text descriptions and the coroner's files for these cases. The latter contained additional detail on the circumstances of injury.

Population-based rates were calculated using Statistics in New Zealand demographic data published annually by the NZHIS ${ }^{28}$. Trends in annual incidence rates were analyzed using the $\chi^{2}$ test for linear trend in proportion ${ }^{29}$.

\section{Results}

A total of 2098 first admissions to public hospitals were identified for the period 1979-1988. The annual incidence rates are shown in Figure 1. These increased from 3.1 per 100000 persons per year $(95 \%$ confidence interval $=2.5-3.7$ ) in 1979 to 9.3 per 100000

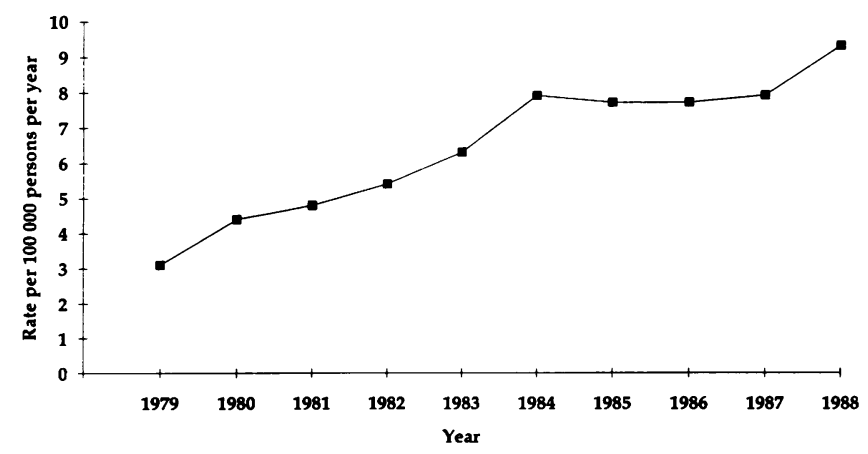

Figure 1. Annual incidence rates for hospitalizations, 1979-1988

persons per year $(\mathrm{CI}=8.3-10.4)$ in 1988 , a threefold increase. A test for linear trend indicated that this increase was statistically significant $\left(P<0.001, \chi^{2}=\right.$ 172.683 , d.f. $=9$ ).

The mean age of victims was 10 years, with $96 \%$ being less than 20 years of age. Table 1 shows the incidence rates by age groups for those under 20 years of age, with the 5-9 year age group having the highest rate of 30.3 per 100000 persons per year $(\mathrm{CI}=$ 28.2-32.4). Among those under 20 years of age, the incidence rate for females was 21.9 per 100000 persons per year $(C I=20.7-23.2)$, which was significantly higher than the rate of 13.9 per 100000 males per year $(\mathrm{CI}=13.0-14.9)$ (Fisher's exact test, 2-tail, $P<0.001)$.

\section{Circumstances}

For $1521(72 \%)$ of the incidents there was sufficient information available to identify the place of occurrence clearly. Of these, $71 \%(n=1087)$ occurred at home, $12 \%(n=180)$ at school, $8 \%(n=126)$ in parks, $5 \%(n=73)$ in public buildings, and $4 \%(n=55)$ in holiday places. The number occurring at home increased from 34 in 1979 to 183 in 1988.

According to the ICD ${ }^{25}$ classification of the external causes of injury (E codes), 1921 of the incidents $(92 \%)$ involved falls (E882-888), with the majority $(n=$ 1857) of these being classified as 'other fall, from one level to another' (E884). One hundred incidents (5\%) involved collisions (E916-917), 59 (3\%) involved over-exertion (E927), 10 involved being caught in or between objects (E918), seven involved sharp objects (E920), and one involved an unspecified environmental cause (E928). Examination of the descriptive information recorded for each case revealed that the most common cause of injury involved the victim

Table 1. Incidence rates for hospitalizations by age group, 1979-1988

\begin{tabular}{llrl}
\hline Age group & Frequency & Rate $^{*}$ & 95\% confidence interval \\
\hline $0-4$ & 247 & 9.7 & \multicolumn{1}{c}{$8.6-11.0$} \\
$5-9$ & 818 & 30.3 & $28.2-32.4$ \\
$10-14$ & 787 & 26.5 & $24.7-28.5$ \\
$15-19$ & 157 & 5.1 & $17.1-18.6$ \\
\hline
\end{tabular}

*Rate per 100000 persons per year 
falling off the trampoline and striking the ground surface or an external object $(80 \%)$, followed by the victim falling onto the bed of the trampoline $(8 \%)$, and the victim striking the frame of the trampoline $(2 \%)$.

There was not enough descriptive information to distinguish reliably between the involvement of mini-trampolines and standard trampolines or between play and gymnastic-quality trampolines; nor was there sufficient information to identify the skill of the trampolinist or the presence of supervision.

\section{Nature of injury}

The frequency distribution of incidents by the nature of injury sustained, using $\mathrm{ICD}^{25}$ categories, is presented in Table 2. The ICD injury categories reflect both the body site and the type of injury. To distinguish between these two dimensions, the cases were reclassified with respect to both body site and injury type. The most common site of injury was the upper limb (53\%), followed by the lower limb (22\%), head $(16 \%)$ and trunk $(7 \%)$. For the remaining cases, no single body site accounted for more than $1 \%$ of the total. The most common category of injury was fractures $(68 \%)$, followed by intracranial injury $(12 \%)$, sprains $(6 \%)$, dislocations $(6 \%)$, contusions $(4 \%)$ and open wounds $(3 \%)$. For the remaining cases, no single category of injury accounted for more than $1 \%$ of the total.

\section{Severity of injury}

The mean length of stay in hospital was 3.4 days. Of the 2098 cases, $886(42 \%)$ were in hospital for 1 day, $361(17 \%)$ for 2 days, and the remainder for 3 days or more.

Application of the ICDMAP programme ${ }^{27}$ to the 1988 data provided AIS $^{26}$ severity scores for the 311 cases occurring in that year. Of these, two $(1 \%)$ were assigned a severity score of ' 4 ' (severe), 33 (11\%) a score of ' 3 ' (serious), $245(79 \%)$ a score of ' 2 ' (moderate), and $29(9 \%)$ a score of ' 1 ' (minor). In two cases the ICDMAP programme was unable to assign a severity score.

Table 2. Nature of injury, $1979-1988$

\begin{tabular}{lrr}
\hline Category* & $n$ & $\%$ \\
\hline Fracture of radius and/or ulna & 591 & 28 \\
Fracture of humerus & 380 & 18 \\
Intracranial injury of other and unspecified nature & 123 & 6 \\
Fracture of ankle & 121 & 6 \\
Concussion & 118 & 6 \\
Fracture of tibia and/or fibula & 117 & 6 \\
Dislocation of elbow & 84 & 4 \\
Sprain or strain of other and unspecified parts of & 63 & 3 \\
back & 43 & 2 \\
Fracture of face bones & 34 & 1 \\
Contusion of trunk & 424 & 20 \\
Other & \\
TOTAL & 2098 & 100 \\
\hline
\end{tabular}

${ }^{*} I C D^{25}$ categories; ${ }^{+}$no single category represented more than $1 \%$ of the total
Since the call for controls on trampoline use has been motivated by reports of severe head and spinal injuries, the nature and circumstances of injuries involving these body sites were examined separately. Among the 311 cases recorded in 1988, there were 33 with head injuries. Of these, two had severe intracranial injuries (one of which was due to falling off the trampoline and the other to striking the frame of the trampoline) and two had serious injuries (one of which involved a fractured skull and was due to falling off the trampoline, while details of the other were not available). The remaining 29 cases were of moderate severity. There were nine cases of spinal injury. Of these, three had serious fractures, two of which were sustained when the victim fell onto the trampoline bed and one when the victim fell off the trampoline and struck the ground surface. Of the remaining cases the injuries were moderate in one case and minor in five.

Injuries to the upper and lower extremities were more common than either head or spinal injuries. In 1988 there were $171(55 \%)$ to the upper extremity and $63(20 \%)$ to the lower extremity. Examination of the former indicated that all $12(7 \%)$ which involved serious injury were fractures sustained when the victim fell off the trampoline. The remaining 159 were of moderate or minor severity. Sixteen $(25 \%)$ of the 63 lower-extremity injuries were serious, with the majority involving fractures resulting from falling off the trampoline. The remaining 46 were of moderate or minor severity and in one case the level of severity was unknown.

Since the international literature suggests that the majority of severe injuries are sustained on landing in the centre of the trampoline bed, the circumstances of the severe and serious injuries were also examined separately. Of the two cases of severe injury recorded in 1988, neither struck the bed of the trampoline. One fell off the trampoline and struck the ground surface and one struck the frame of the trampoline. Of the 33 serious cases recorded in that year, four fell onto the bed, 25 fell off and four were injured in other circumstances.

\section{Mortality}

Examination of the HIS mortality files for the period 1978-1987 identified two cases of death due to injuries sustained on a trampoline. Examination of the coroner's files for these cases indicated that both victims were under the age of 20 years, disabled, in residential care and unsupervised at the time of the incident. Both are believed to have struck the frame of the trampoline.

\section{Discussion}

A threefold increase in the incidence of trampolinerelated hospitalizations was identified for the 10-year period under investigation. Being based on total population figures, the reported rates do not take into account exposure to trampolines in terms of either availability or use, factors which may be responsible for the increasing rate. While it has been estimated that there are 40000 trampolines in regular use in 
New Zealand ${ }^{20}$, trampolinists registered through trampolining clubs numbered only 267 in 1989 and 270 in $1990^{30}$. No figures are available on the use of trampolines outside of registered clubs. Given the widespread recreational use of trampolines, rates based on registered participants only would be misleading.

Those in the past who have called for bans on trampoline use have focused on reported cases of severe head and neck injuries, generally sustained on impact with the bed of the trampoline. In the present study, however, it was found that there was a low incidence of severe head and spinal injuries, with the majority of injuries being to the upper and lower extremities. Moreover, in the great majority of incidents the victims fell from the trampoline and sustained injury on impact with the surface below. A number of measures could be taken to reduce both the risk of falling from the trampoline and of being injured on impact with the surrounding surface, including:

1. Ensuring that the bed is of adequate size to permit rebounding without falling off the trampoline in the event of a loss of balance or control;

2. Ensuring that an adequate number of competent 'spotters' are in position whenever the trampoline is in use;

3. Ensuring that in siting the trampoline, allowance is made for a minimum area of unobstructed space surrounding it (cf. the operating area requirements for playground equipment ${ }^{31}$ );

4. Ensuring that the surfaces surrounding the trampoline have impact-absorbing characteristics (cf. the surfacing rquirement for playground equipment $^{31}$ );

5. Siting the trampoline in a pit so that the bed is level with the ground surface;

6. Ensuring that correct techniques are used for mounting and dismounting.

A further measure to reduce the risk of falling when mounting or dismounting from the trampoline would be to attach a vertical rung ladder to the trampoline frame. One reservation about this measure, however, is that it may enable younger children to gain access to the trampoline bed without the assistance of an older person and thereby increase the exposure of this group. Given this reservation, careful consideration of this measure is required.

Chalmers and Langley ${ }^{32}$, in their study of hospitalizations associated with falls from playground equipment in children aged less than 15 years, found that $59 \%$ of trampoline injuries occurred at home. In the present study, which included victims of all ages, it was found that over the 10 -year period $71 \%$ of incidents occurred at home. While this high proportion of injuries occurring at home might simply be a function of exposure, it may relate also to factors such as poor-quality equipment (e.g. inadequate bed size or lack of safety pads), bad siting (e.g. proximity to obstacles or concrete), misuse (e.g. multiple users on bed), and lack of supervision (e.g. lack of 'spotters'). While the measures described above would address a number of these issues, the following additional measures could be taken:
1. Ensuring that frame padding is securely attached to the frame so as to cover all portions of the top surface of the frame, the outer hooks of the trampoline springs, the spring-anchor devices on the frame, and the springs;

2. Ensuring that frame padding is supplied by the manufacturer as an integral part of the trampoline and not as an optional extra;

3. Ensuring that there is only one user on the trampoline bed at any time.

On the basis of the findings reported here, and recognizing the popularity of trampolining in New Zealand, we are unable to support the notion of a total ban on the use of the trampoline. Nevertheless, we accept the argument made in the international literature that trampolining should be treated as a potentially dangerous activity and that controls are necessary to reduce the frequency and severity of injuries associated with this equipment. We therefore recommend that:

1. The trampoline is not provided as 'play equipment' in any setting, including the home;

2. The trampoline is used only under expert instruction or supervision;

3. Disabled children should not use the trampoline except under constant supervision;

4. Initiatives taken to develop guidelines for competitive trampolining-and in particular the training of accredited instructors, supervisors and coaches - should be supported by national, regional and local organizations concerned with the promotion and control of sporting and recreational activity.

While ASTMF381-84 ${ }^{1}$ and NZS 5855:1993 ${ }^{23}$ together address many of the issues raised by the above findings and are reflected in the above recommendations, we believe that further consideration must be given to measures that will reduce the impact of falls from the trampoline. We refer in particular to the recommendation made above for the provision of impact-absorbing surfaces surrounding trampolines. Finally, we do not consider it sufficient to require use of the trampoline 'only with mature, knowledgeable supervision' (NZS 5855:1993:6.4.3(10) ${ }^{23}$ ). We believe that in the absence of information on exposure in different settings, a conservative stance is warranted. In addition to the above requirement, therefore, standards should clearly state that trampolines are not to be provided for use as 'play equipment' in any setting, including the home. It is most unlikely that in this latter setting, knowledgeable supervision and an adequate number of competent 'spotters' would be available at all times during which the trampoline was in use.

\section{Acknowledgements}

This project was funded by a grant from the Accident Rehabilitation and Compensation Insurance Corporation of New Zealand (ACC). The Injury Prevention Research Unit is funded jointly by the ACC and the Health Research Council of New Zealand. The authors are grateful to the New Zealand Health Information Service for the provision of data; to the Director General of Health for approval to publish this paper; to Stephen Marshall, Biostatistician 
with the Injury Prevention Research Unit, for his assistance with the data analysis; and to Dr John Langley and Dr Ann Isaac for their comments on earlier versions of this paper. The opinions expressed in this paper are those of the authors and are not necessarily those of the above organizations or individuals.

\section{References}

1 American Society for Testing and Materials. Standard Consumer Safety Specification for Components, Assembly, and Use of a Trampoline (ASTM F381-84). Philadelphia: American Society for Testing and Materials, 1984.

2 Hammer A, Schwartzbach AL, Paulev PE. Trampoline training injuries: one hundred and ninety-five cases. $\mathrm{Br}$ Sports Med 1981; 15: 151-8.

3 Rapp GF, Nicely PG. Trampoline injuries. Am J Sports Med 1978; 6: 269-71.

4 Torg JS, Das M. Trampoline-related quadriplegia: review of literature and reflections on the American Academy of Pediatrics, position statement. Pediatrics 1984; 74: 804-12.

5 American Academy of Pediatrics. Committee on Accident and Poison Prevention: Policy Statement: Trampolines. Evanston, Illinois: American Academy of Pediatrics, 1977.

6 Hammer A, Schwartzbach AL, Paulev PE. Some risk factors in trampolining illustrated by six serious injuries. $\mathrm{Br} J$ Sports Med 1982; 16: 27-32.

7 Woodward GA, Furnival R, Schunk JE. Trampolines revisited: a review of 114 pediatric recreational trampoline injuries. Pediatrics 1993; 89: 849-54.

8 Lascari A. Trampoline safety. CAHPER Journal 1980; 46: 9-11.

9 Meredith MM. Don't sell your trampoline. J Phys Educ Rec and Dance 1981; 52: 82-3.

10 Rosenthal A. The trampoline: fitness boon or safety hazard? Today's Health 1969; 33-35.

11 American Academy of Pediatrics. Committee on Accident and Poison Prevention and Committee on Pediatric Aspects of Physical Fitness, Recreation, and Sports: Policy Statement: Trampolines II. Pediatrics 1981; 67: 438-9.

12 American Alliance for Health, Physical Education and Recreation. Use of the trampoline for the development of competitive skills in sports. Use of trampolines and minitrampolines in physical education. AAHPER position statements on trampolines. J Phys Educ and Rec 1978; 49: 14

13 Benis HG. Trampoline Safety. Wellington: Accident Compensation Corporation, 1983.

14 Canadian Alliance for Health, Physical Education, and Recreation. Use of trampoline and mini-trampolines in P.E (Position Statement.) CAHPER Times 1978; 3: 2-3.
15 Hennessy JT. Safety awareness and trampoline skills. J Phys Educ and Rec 1980; 51: 36-8.

16 Hodge J. Trampolining safety. Part 2. Safety in learning on the trampoline. CAHPER Journal 1980; 46; 19-23.

17 Canadian Gymnastics Federation. Trampoline Coaching Certification and Safety Manual. Level 1. Ottawa: Canadian Gymnastics Federation, 1979.

18 Isaac A. Coaching Scheme. Auckland: New Zealand Trampoline Association, 1991.

19 Johnson A, Ross D. Trampoline Coaching Certification and Safety Manual: Level 2. Ottawa: Canadian Gymnastics Federation, 1984.

20 Wilson BD, Chalmers DJ, Hume PA. The rise and fall of the trampoline: a review of trampoline injury literature. In preparation.

21 Benis HG. Trampoline Safety Technical Report No. 1, Sections 1-3. NZ J Sports Med 1980; 8: 16-21.

22 Chalmers DJ, Langley JD. Epidemiology of playground injuries resulting in hospitalisation. J Paed Child Health 1990; 26: 329-34.

23 Standards Association of New Zealand. New Zealand Standard: Consumer Safety Specification for Components, Assembly, and Use of a Trampoline (NZS5855:1993). Wellington: Standards Association of New Zealand, 1993.

24 Hume PA, Chalmers DJ, Wilson BD. Trampolines: a health risk? In preparation

25 World Health Organization. International Classification of Diseases, 1975 Revision. Geneva: World Health Organization 1977.

26 Association for the Advancement of Automotive Medicine. The Abbreviated Injury Scale. Des Plaines: Association for the Advancement of Automotive Medicine, 1990.

27 McKenzie EJ, Shapiro S, Moody M, Siegal JH, Smith RT. Predicting post trauma functional disability for individuals without severe brain injury. Medical Care 1986; 24: 377-87.

28 National Health Statistics Centre. Mortality and Demographic Data 1987. Wellington: Department of Health. 1988.

29 Snedecor GW, Cochrane WG. Statistical Methods, 7th edn. Ames, Iowa: Iowa State University Press, 1980.

30 Hillary Commission for Sport Fitness and Leisure. Personal communication, 1991.

31 Standards Association of New Zealand. New Zealand Standard Specification for Playgrounds and Playground Equipment (NZS5828). Wellington: Standards Association of New Zealand, 1986

32 Chalmers DJ, Langley J. Childhood falls from playground equipment resulting in admission to hospital: descriptive epidemiology. In Sime JD (ed) Safety in the Built Environment. London: Spon, 1988. 